\title{
The Outcome of Vaginal Birth after One Caesarean Section (VBAC)-A Descriptive Study
}

\author{
Dr. Mst. Jesmin Akter ${ }^{1 *}$, Dr. Eliza Shirin ${ }^{2}$ \\ ${ }^{1}$ Assistant Professor, Department of Gynecology \& Obstetrics, Cumilla Medical College \& Hospital, Cumilla, Bangladesh \\ ${ }^{2}$ Specialist, Department of Gynecology \& Obstetrics, Cumilla Medical College \& Hospital, Cumilla, Bangladesh
}

\begin{abstract}
DOI: $10.36348 /$ sijog.2020.v03i12.003
| Received: 21.11.2020 | Accepted: 03.12.2020 | Published: 10.12.2020
\end{abstract}

*Corresponding author: Dr. Mst. Jesmin Akter

\section{Abstract}

Background: Caesarean section has become the most performed major operation in obstetrics. The increasing rate of primary caesareans section becomes high worldwide due to early detection of fetal and maternal complications. Repeated caesarean section is one of the major contributory factors for increasing this rate very significantly. Now a day, vaginal delivery of pregnant mothers with the history of previous one caesarean with non-recurrent cause was established. It has been shown that the outcome of trial of labor in past caesarean delivery is acceptable, effective and safe for both mother and fetus, if the women are properly selected. Objective: The objectives of this study was to determine the outcomes of vaginal birth after caesarean section (VBAC) in case of previous one caesarean section to reduce the subsequence cesarean section with its complication. Materials and Methods: It was a cross sectional study carried out in the Maternity Unite-1, Department of Gynecology \& Obstetrics, Sir Salimullah Medical College and Mitford hospital, Dhaka, Bangladesh, held on January 2010 to December 2010. Out of total 380 admitted pregnant women who had previous one caesarean section, 50 pregnant women of 37-42 weeks of gestational age with the history of one caesarean delivery with alive baby were selected as study population following consecutive and purposive sampling method. Patients with spontaneous onset labor but preterm pregnancy with any contraindication or prior caesarean section due to recurrent causes, history of classical caesarean section, more than one caesarean section, multiple pregnancy, pregnancy with medical disorder were excluded in the study. Results: Out of total 50 sampled pregnant women, vaginal delivery were done $16(32 \%)$ \& emergency cesarean section were done 34(68\%). According to the age group both vaginal \& cesarean section 20-30 years were predominant, which were 8(50\%) and 17(50\%) respectively. Regarding antenatal care $13(81.25 \%)$ of vaginal delivery cases were regular. On the other hand, only $10(29.41 \%)$ of cesarean section were regular in care. Fetal survival outcome in vaginal \& caesarean which were 14(87.5\%) and 33(97.05\%) respectively. Comparing the maternal complication maximum number of vaginal delivery group had no complications. Conclusion: In this study $14.5 \%$ pregnant women went spontaneous labor. By permitting a trial of labor in our pregnant mother with a previous caesarean section, we will be able to reduce the caesarean delivery rate. Women for VBAC needs regular antenatal checkup, timely admit in hospital and delivery under close supervision. Therefore, all pregnant women with prior delivery by caesarean section need not necessarily be delivered by caesarean section during the next pregnancy of a pregnant women.

Keywords: Caesarean section, vaginal section, VBAC.

Copyright () 2020 The Author(s): This is an open-access article distributed under the terms of the Creative Commons Attribution 4.0 International License (CC BY-NC 4.0) which permits unrestricted use, distribution, and reproduction in any medium for non-commercial use provided the original author and source are credited.

\section{INTRODUCTION}

Caesarean section had been a part of human culture since ancient times and there are histories in both Western and Eastern cultures of this procedure resulting in live mothers and off mechanisms. Numerous references to caesarean section appear in ancient Hindu, Egyptians, Greecians, Romans and other
European folklore [1]. In past 20 years, the rate of CS has steadily increased from about $5 \%$ to more than $20 \%$ [2]. The policy- once a caesarean always a caesarean is no longer rational. A planned vaginal birth after a previous CS should be recommended for women whose first CS was by lower segment transverse incision and who have no other indication for CS in present pregnancy $[3,4]$. Delivery in post-caesarean is a matter 
of debate. The Craigin's dictum "Once a caesarean, always caesarean" no longer valid today [5]. Because the caesarean rate in that time was only $1-2 \%$ and were mostly classical type. Perhaps the first written record of cesarean section was of surviving a mother and baby of Switzerland in 1500, Jacob Nufer, performed the operation on his wife [6]. Successful cesarean section accomplished by native healers in Kahura, Uganda [6]. As early as 1876, Italian professor Eduardo Porro had promoted hysterectomy in harmony with cesareans to control uterine hemorrhage and avert systemic infection. This enabled him to reduce the incidence of post-operative sepsis. But his disfiguring amplification on cesarean section was soon precluded by the employment of uterine sutures. In 1882, Max Saumlnger, of Leipzig made such a strong case for uterine sutures that surgeons commenced to change their practice. The silver wire stitches he endorsed were themselves new, having been developed by America's premier nineteenth-century gynecologist J. Marion Sims. Sims had invented his sutures to treat the vaginal tears (fistulas) that resulted from traumatic childbirth.6Between 1880 and 1925, obstetricians experimented with transverse incisions in the lower segment of the uterus. This refinement reduced the risk of infection and of subsequent uterine rupture in pregnancy. An advance modification-vaginal cesarean section-helped avoid peritonitis in patients who were already suffering from certain infections. The need for that form of section, however, was virtually eliminated in the post-World War II period by the development of modern antibiotics. Penicillin was discovered by Alexander Fleming in 1928 and, after it was purified as a drug in 1940, became generally available and dramatically reduced maternal mortality for both normal and cesarean section births [6]. Since 1940, the trend toward medically managed pregnancy and childbirth has steadily accelerated. Many new hospitals were built in which women gave birth and in which obstetrical operations were performed. By 1938, approximately half of U.S. births were taking place in hospitals. By 1955, this had risen to ninety-nine percent [6]. In 1970, the cesarean section rate was about $5 \%$; by 1988 , it had peaked at $24.7 \%$. In 1990 , it had decreased slightly to $23.5 \%$, primarily because more women were attempting vaginal births after cesarean deliveries [6]. In the United States almost one quarter of all babies are now delivered by cesarean section - approximately 982,000 babies in 1990.6Perhaps one of the most important factors is the changing opinion toward the formula "once a cesarean section, always a cesarean section." This expression embodied the notion that once a woman had a cesarean she would require surgery for all subsequent deliveries. This was, apparently, the cause of the greatest increase in cesarean sections between 1980 and $1985 .^{6}$ The American College of Obstetricians and Gynecologists responded swiftly to calls from within the organization and from the patient population and in 1982, as a standard of care, recommended a trial of labor in selected cases of prior cesarean section. In 1988, the guidelines were expanded to include more women with previous cesarean births. Hence, there was a steady increase in vaginal births after cesarean in the late 1980's. In 1990, an estimated 90,000 women gave birth vaginally after cesarean section [6]. In 1980s, the National Institute of Health Consensus Conference in USA recommended that the otherwise in uncomplicated pregnant women with a prior lower segment transverse caesarean incision, the patients be encouraged to under a trial of labor [7]. In early caesarean section, no suture materials were placed in uterus. Therefore, hemorrhage and sepsis were the cause of death. During 1980 to 1990s, many healthcare community and management care organization adopted the policy of attempting the trial labor in women with history of previous caesarean section, even in the face of unacceptable risk to the mother and to the fetal. From that initiatives, this practice is known as Vaginal Birth After Caesarean (VBAC). An attempted of vaginal birth is preferred method of delivery management for most patients in whom the primary caesarean section was performed for non-recurring causes, e.g. fetal distress, cephalopelvic disproportion (CPD) and non-progress of labor [8]. In a study in the UK, 7065 of women undergoing VBAC had a history of previous vaginal delivery. These women achieved a high rate of VBAC success $(86.6 \%)$ compared with only $60.9 \%$ in women without a history of previous vaginal delivery [9]. Attempting VBAC decrease the risks associated with the surgical delivery. But VBAC attempts are not risksfree. Even among the best candidates, the risk of uterine rupture is between 0.2 to $1.5 \%$. Impending rupture or wound dehiscence along with the old scar usually not so much life threatening for mother and babies. However, rupture uterus is significant cause of maternal morbidity, mortality and fatal death. When VBAC attempts failed that increase significant risk of infection both mother and baby [10]. Without knowing the type of previous scar, it is very much difficult to give decision about the mode of the delivery. So, attempting VBAC with unknown uterine scar, there is a significant increased risk of uterine rupture and subsequent maternal and perinatal death. A skilled sonographer has the ability to identify the scar, its direction, thickness and its abnormalities if any existed. It was found that the thickness of the lower uterine segment decreases from $6.7 \mathrm{~mm}$ to $3 \mathrm{~mm}$ at the time of delivery, however it decreases to $2.3 \mathrm{~mm}$ in patients with previous caesarean section. The women with thickness of lower uterine segment $<2 \mathrm{~mm}$ had intrapartum uterine rupture [11]. There is a definite risk of uterine rupture in vaginal birth after caesarean delivery (VBAC) often leading to disasters which can be avoided by rapid diagnosis and prompt intervention. Evidence confirming the safety of VBAC within proper guidelines has been available for more than 10 years [12-16]. However, wide variations in VBAC rates still exist between hospitals and physicians. The present study was undertaken to re-ascertain these facts with the hope that more women will be encouraged to avoid 
an unnecessary repeat caesarean section by opting for vaginal delivery. VBAC offers individual advantages over a repeat caesarean section since the operative morbidity and mortality are completely eliminated, the hospital stay is much shorter and expenses involved are much less [15, 17-19]. The rate of caesarean section needs to be reduced and this can be achieved to a small extent by avoiding primary caesarean sections done without explicit indications and more importantly by resorting to a trial of vaginal delivery after previous caesarean section which is safe for the fetus [20-23]. The purpose of this study was to evaluate the efficacy and safety of VBAC. So, successful VBAC reduced the incident of post-partum morbidity, infection, blood transfusion and hospital stay. Therefore, all women with prior delivery by cesarean section need not necessarily be delivered by caesarean section during there next pregnancy.

\section{MATERIALS AND METHODS}

This cross section study was conducted in the Department of Obstetrics and Gynecology, Sir Salimullah Medical College \& Mitford Hospital, Dhaka, Bangladesh, from January 2010 to December 2010. A total 380 patients were admitted as term pregnancy with the history of one caesarean section. Out of 380 admitted pregnant women, consecutive 50 patients were purposively selected, who fulfilled the inclusion and exclusion criteria as sample population. Patients with spontaneous onset labor but preterm pregnancy with any contraindication or prior caesarean section due to recurrent causes like cephalopelvic disproportion, major degree placenta praevia, transverse lie, history of classical caesarean section, associated medical disorder like anemia $(\mathrm{Hb}<10 \mathrm{gm} \%)$, pregnancy include hypertension, diabetes, heart disease, chronic renal disease, breech presentation, history of postoperative wound infection following previous LSCS, postdated pregnancy with unfavorable cervix, estimated fetal weight $>3.5 \mathrm{~kg}$, more than one caesarean section and multiple pregnancy were excluded in the study. A protocol was designed including details history of the patients were observed from admission. Whether labor was spontaneous or induced it was monitored by hourly recording of vital parameters i.e. temperature, pulse, respiration, $\mathrm{BP}$, continuous electronic fetal monitoring by cardiotocography, petrography, uterine monitoring contractions, close watch for the early recognition of scar dehiscence by identifying maternal tachycardia in absence of fever, vaginal bleeding, scar tenderness and fetal heart rate alterations. Attempt at vaginal delivery was abandoned if there was any suspicion of scar dehiscence or sign of fetal distress or unsatisfactory progress of labor. Vacuum extraction was used to cut short the second stage. Rates of repeat CS, frequency of maternal variables (residence, ethnicity, gravidity etc.) indications of CS and fetomaternal outcome are expressed in percentage. Statistical analysis performed with SPSS version 20.0

\section{RESULTS}

Total 50 women met the study criteria. Out of 50 , vaginal delivery was done 16 pregnant women and caesarean section were done 34 pregnant mothers.

Table-1 shown that in vaginal and caesarean section patients age group 20- 30 years were predominant. Regarding parity, 1 was highest $8(50 \%)$ of vaginal delivery cases, whereas $1 \& 2$ was highest $15(44 \%)$ of caesarean cases. In gravida, $2^{\text {nd }}$ issue was more in vaginal delivery cases and $3^{\text {rd }}$ issue was in caesarean section. Both parity $(\mathrm{p}=<0.01)$ and gravida's $(\mathrm{p}=<0.05) \mathrm{p}$ values were significant.

Table-1: Patients Profile of the Study $(\mathrm{N}=50)$

\begin{tabular}{|c|c|c|c|}
\hline Parameters & $\begin{array}{l}\text { V D } \\
n=16(\%)\end{array}$ & $\begin{array}{l}\text { CS } \\
n=34(\%)\end{array}$ & p-Value \\
\hline \multicolumn{4}{|l|}{ Age (years) } \\
\hline 20-30 years & $8(50.0)$ & $17(50.0)$ & \multirow[t]{3}{*}{$0.730^{\mathrm{ns}}$} \\
\hline $31-40$ years & $6(37.5)$ & $14(41.18)$ & \\
\hline$>40$ years & $2(12.5)$ & $3(8.82)$ & \\
\hline \multicolumn{4}{|l|}{ Parity } \\
\hline 1 & $8(50.0)$ & $15(44.0)$ & \multirow[t]{3}{*}{$<0.01^{\mathrm{s}}$} \\
\hline 2 & $6(37.5)$ & $15(44.0)$ & \\
\hline 3 & $2(12.5)$ & $4(12.0)$ & \\
\hline \multicolumn{4}{|l|}{ Gravida } \\
\hline $2^{\text {nd }}$ & $8(50.0)$ & $14(41.0)$ & \multirow[t]{3}{*}{$<0.05^{\mathrm{ns}}$} \\
\hline $3^{\text {rd }}$ & $5(31.0)$ & $18(53.0)$ & \\
\hline $4^{\text {th }}$ or more & $3(19.0)$ & $2(6.0)$ & \\
\hline
\end{tabular}




\section{Patients Age Group Wise Distribution}

\section{0}

50

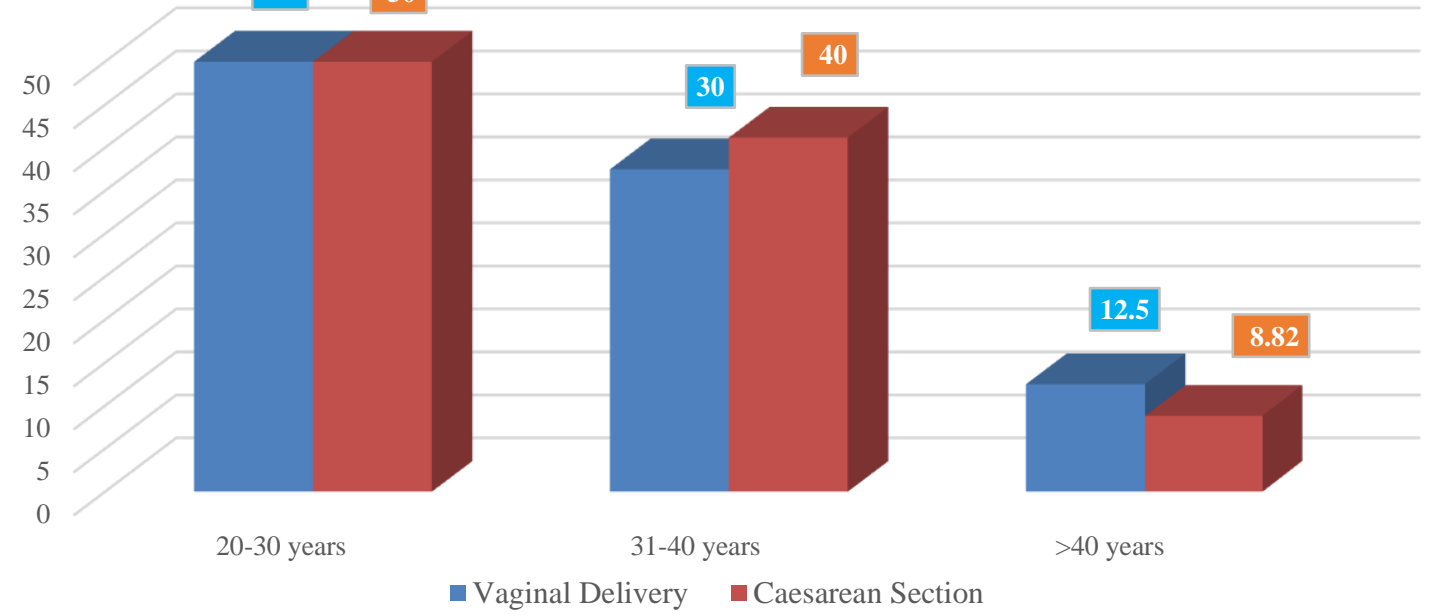

Fig-1: Patients Age Wise Group Distribution

Table-2 shown that out of total 50 pregnant women caesarean section was done $34(68 \%)$ and vaginal delivery issue was $16(32 \%)$. $\mathrm{P}$ value was in significant $(\mathrm{p}=<0.001)$.

Table-2: Mode of Delivery Done of Pregnant Women $(\mathbf{N}=50)$

\begin{tabular}{|l|l|l|l|}
\hline Mode of Delivery & $\mathbf{n}$ & $\mathbf{\%}$ & p-Value \\
\hline Vaginal Delivery & 16 & 32.0 & $<0.001^{\text {s }}$ \\
\cline { 1 - 3 } Caesarean Section & 34 & 68.0 & \\
\hline Base & 50 & 100.0 & \\
\hline
\end{tabular}

\section{Mode of Delivery of Pregnant Women}

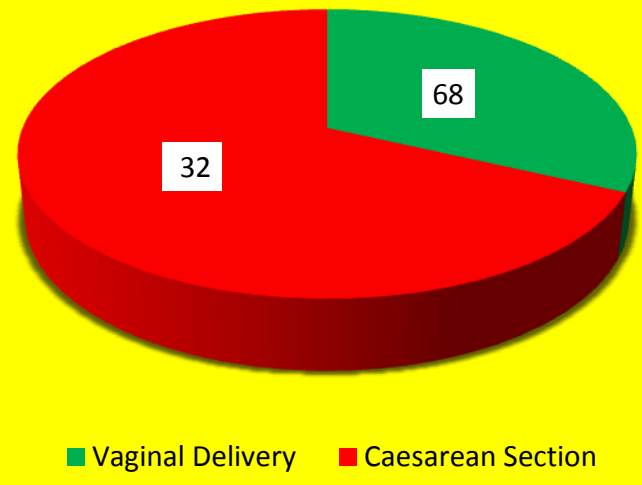

Fig-2: Mode of Delivery of Pregnant Women

Table-3 shown that, regarding regular antenatal care issue of the sample pregnant women, in vaginal deliver cases was higher in percentage $81.25 \%$, on the other hand it was only $29.41 \%$ in caesarean section cases. Mean value was Mean value $35.29( \pm 9.37)$ which significant of $p$ value $\left(p=<0.001^{s}\right)$.

Table-3: Antenatal Care with Mode of Delivery Done $(\mathrm{N}=50)$

\begin{tabular}{|l|l|l|l|}
\hline Parameters & $\begin{array}{l}\text { VD } \\
\mathbf{n = 1 6}(\boldsymbol{\%})\end{array}$ & $\begin{array}{l}\text { CS } \\
\mathbf{n = 3 4}(\boldsymbol{\%})\end{array}$ & \multirow{2}{*}{$<0.001^{\mathrm{s}}$} \\
\hline Regular & $13(81.25)$ & $10(29.41)$ & \\
\hline Irregular & $3(18.75)$ & $21(61.76)$ & \\
\hline None & $0(0.0)$ & $3(8.82)$ & \\
\hline Base & 14 & 34 & \\
\hline
\end{tabular}


Table-4 shown that, comparison labor pain between vaginal delivery and caesarean section the mean value was $4.11 \pm 1.92(\mathrm{p}=0.345)$ and $7.17 \pm 2.42$ $(\mathrm{p}=<0.001)$ respectively.

Table-4: Comparison between Vaginal Delivery and Caesarean Section $(\mathbf{N}=\mathbf{5 0})$

\begin{tabular}{|l|l|l|l|}
\hline Parameters & $\begin{array}{l}\text { VD } \\
\mathbf{n = 1 6}(\%)\end{array}$ & $\begin{array}{l}\text { CS } \\
\mathbf{n = 3 4}(\%)\end{array}$ & p-Value \\
\hline Clinical Presentation & & & \\
\hline Presence with labor pain & $12(75.0)$ & $24(71.0)$ & \multirow{2}{*}{$0.345^{\mathrm{ns}}$} \\
\hline Presence without labor pain & $4(25.0)$ & $24(71.0)$ & \\
\hline Interval between previous \& present pregnancy(yrs.) & $4.11 \pm 1.92$ & $7.17 \pm 2.42$ & $<0.001^{\mathrm{s}}$ \\
\hline
\end{tabular}

Table-5 shown that, regarding fatal outcome, a live fatal was significantly higher $97.05 \%$ than vaginal deliver cases $87.5 \%$. In neonatal death issue 1 baby died in vaginal delivery case, but in caesarean section it was nil in number.

Table-5: Fetal Outcomes ( $N=50)$

\begin{tabular}{|l|l|l|l|}
\hline Parameters & $\begin{array}{l}\text { VD } \\
\mathbf{n = 1 6}(\boldsymbol{\%})\end{array}$ & $\begin{array}{l}\mathbf{C S} \\
\mathbf{n}=\mathbf{3 4}(\boldsymbol{\%})\end{array}$ & $\mathbf{p}-$ Value \\
\hline A live fetal & $14(87.5)$ & $33(97.05)$ & $0.83^{\text {ns }}$ \\
\hline Still birth & $1(6.25)$ & $1(6.2)$ & $0.05^{\text {ns }}$ \\
\hline Neonatal death & $1(6.25)$ & $0(0.0)$ & $0.481^{\text {ns }}$ \\
\hline Resuscetation Required & $5(31.25)$ & $5(14.7)$ & $0.359^{\text {ns }}$ \\
\hline
\end{tabular}

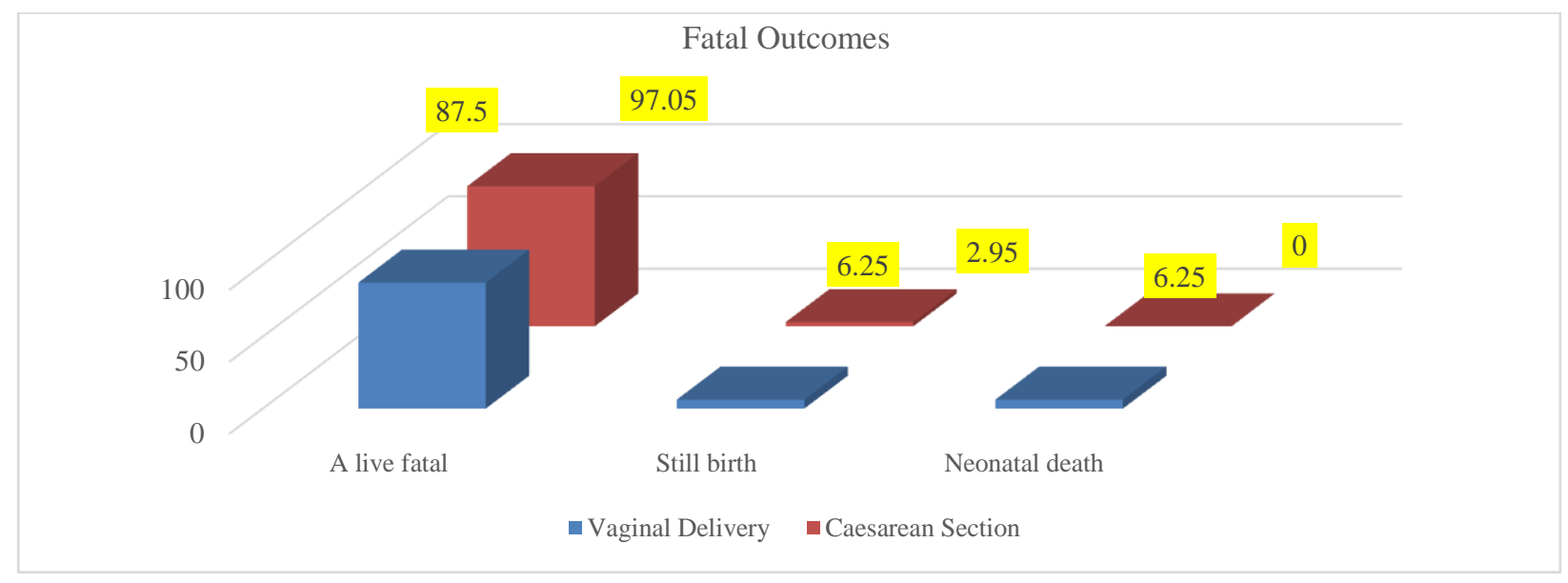

Fig-3: Fetal Outcomes

Table-6 shown that, both vaginal delivery and caesarean section mean score was higher in APGAR (appearance, pulse, grimace, activity, respiration of fetal) score in 5 minute than 1 minute. Fetal weight was significantly higher vaginal delivery than caesarean section.

Table-6: Fetal APGAR Score \& Weight $(\mathbf{N}=50)$

\begin{tabular}{|l|l|l|l|}
\hline Fetal Parameters & $\begin{array}{l}\text { VD } \\
\mathbf{n = 1 6}(\boldsymbol{\%})\end{array}$ & $\begin{array}{l}\text { CS } \\
\mathbf{n = 3 4}(\boldsymbol{\%})\end{array}$ & $\mathbf{p}$-Value \\
\hline APGAR Score-1 minute & $5.57 \pm 1.03$ & $5.12 \pm 0.97$ & $0.052^{\text {ns }}$ \\
\hline APGAR Score-5 minute & $7.43 \pm 1.07$ & $6.98 \pm 1.02$ & $0.065^{\mathrm{ns}}$ \\
\hline Fetal weight $(\mathrm{gm})$ & $2850 \pm 360$ & $2840 \pm 490$ & $0.907^{\mathrm{ns}}$ \\
\hline
\end{tabular}

Table-7 shown that, regarding maternal complications of vaginal delivery PPH (post-partum hemorrhage) was high $18.75 \%$ and of caesarean section, wound infection was $11.76 \%$. Which observed significantly higher than others maternal complications. 
Table-7: Maternal Complications Associated with Mode of Delivery $(\mathrm{N}=50)$

\begin{tabular}{|c|c|c|c|}
\hline Maternal complications & $\begin{array}{l}\text { VD } \\
n=16(\%)\end{array}$ & $\begin{array}{l}\text { CS } \\
n=34(\%)\end{array}$ & p-Value \\
\hline Spinal headache & $0(0.0)$ & $2(5.88)$ & \multirow[t]{7}{*}{$0.189^{\mathrm{ns}}$} \\
\hline Rupture uterus & $1(6.25)$ & $1(2.97)$ & \\
\hline Perineal tear & $1(6.25)$ & $0(0.0)$ & \\
\hline $\mathrm{PPH}$ & $3(18.75)$ & $4(11.76)$ & \\
\hline Hysterectomy & $1(6.25)$ & $1(2.94)$ & \\
\hline Wound infection & $0(0.0)$ & $5(14.21)$ & \\
\hline Wound dehiscence & $0(0.0)$ & $2(5.88)$ & \\
\hline
\end{tabular}

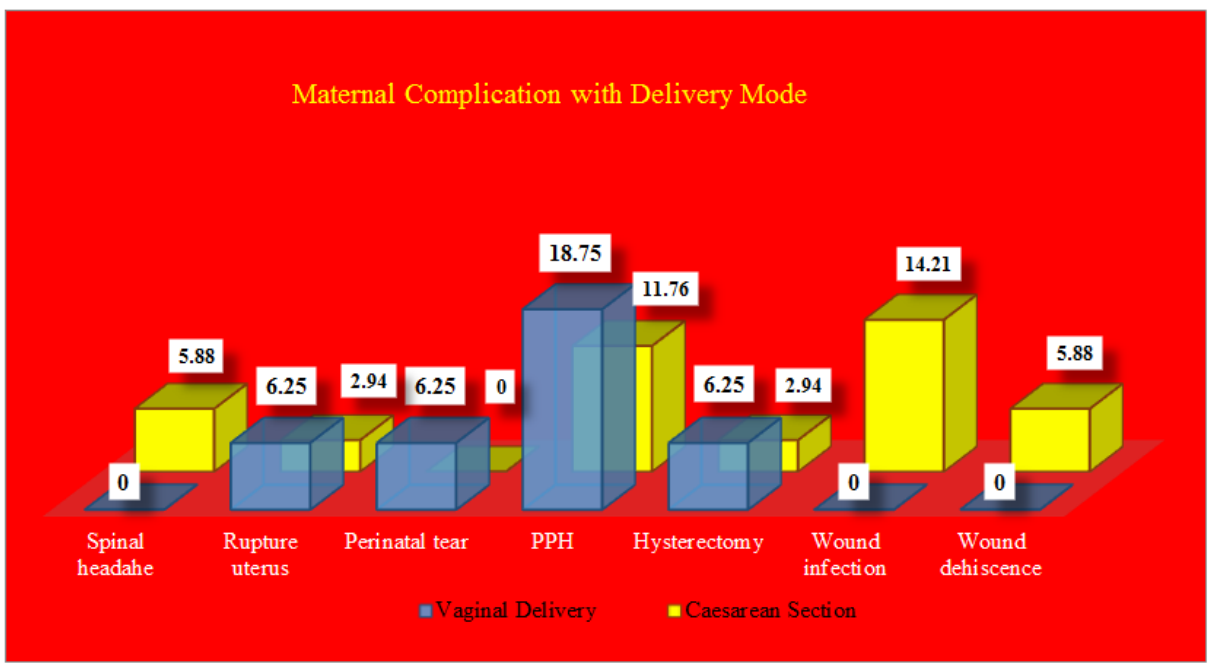

Fig-4: Maternal Complications with Delivery Mode

\section{DISCUSSION}

Caesarean section has become the most performed major operation in obstetrics. The increasing rate of primary caesarean section is due to early detection of fetal and maternal complication. Repeat caesarean section is one of the major contributory factors for increasing rate VBAC. It accounts for one third of all cesarean deliveries. In recent years, there has been increasing concern about the increase in morbidity associated with trial of labor after previous cesarean, particularly the risk of uterine rupture [24]. Despite the known factors which affect the outcome of VBAC like interval between previous cesarean and current pregnancy, indication of previous cesarean, previous successful vaginal deliveries, postoperative wound sepsis, etc. Therefore, reduction in the rate of repeat cesarean section will lead to decrease in cesarean section rate. Hence, the importance of more patients being allowed to attempt vaginal birth after cesarean (VBAC) is explained. There is no consensus regarding decision of mode of delivery in patients with previous cesarean section. According to the latest data from 150 countries, currently $18.6 \%$ of all births occur by CS, ranging from $6 \%$ to $27.2 \%$ in the least and most developed regions, respectively. Latin American and the Caribbean region has the highest CS rates (40.5\%), followed by Northern America (32.3\%), Oceania $(31.1 \%)$, Europe $(25 \%)$, Asia (19.2\%) and Africa $(7.3 \%)$. Based on the data from 121 countries, the trend analysis showed that between 1990 and 2014, the global average CS rate increased $12.4 \%$ (from $6.7 \%$ to $19.1 \%$ ) with an average annual rate of increase of $4.4 \%$. The largest absolute increases occurred in Latin America and the Caribbean (19.4\%), from $22.8 \%$ 42.2\%), followed by Asian (15.1\%, from $4.4 \%$ to $19.5 \%$ ), Oceania $(14.1 \%, 18.5 \%$ to $32.6 \%)$, Europe $(13.8 \%$, from $11.2 \%$ to $25 \%$ ), Northern America (10\%, from $22.3 \%$ to $32.3 \%$ ) and Africa (4.5\%, from $2.9 \%$ to $7.4 \%)$. Asia and Northern America were the regions with the highest and lowest average annual rate of increase (6.4\% and $6 \%$, respectively) [25].

Patients with prior caesarean delivery needs special management both antenatal and in labor and delivery. We know that many women can safely and successfully have a vaginal birth after caesarean delivery. Current medical evidence indicates that 60$80 \%$ of women can achieve a vaginal delivery following a previous lower uterine segment caesarean delivery [26]. Looking at the rates separately for elective and emergency sections, these rates have increased almost in parallel with each other, the ratio of emergency to elective sections staying roughly at about $60: 40$. The rate of elective caesarean section rose from $5.8 \%$ to $10.6 \%$ in 1999 , a total rise of $83 \%$ [27]. The decrease in women with a previous caesarean section undergoing a trial of labor reflects patient's choice as much as obstetrician's decision. The way in which a woman is counseled will influence this choice. If a doctor, has no 
objections to a repeat caesarean section and informs the woman that her chances of a repeat operation is around $30 \%$ [28], the woman herself will be influenced by this. Evidence suggests that there is significantly greater morbidity associated with a trial of labor compared with an elective caesarean section which will further affect the decision [29]. Maternal request for elective caesarean section must be one of the few instance when the patient can request major surgery with all the inherent risks with no proven benefit to her or her baby. It is surprising that women will choose to subject themselves to a major surgical procedure with all the inherent risks with no proven benefit to their baby or themselves. It has been assumed that this is in fact obstetrician-driven, that women have detected during consultations that obstetricians feel the elective caesarean section is best and have thus requested this. ${ }^{30}$ In this study primarily 380 women were admitted with previous one caesarean section, where elective caesarean section was performed in 212(55.9\%), which correspondents Tongson's study [31]. Tongsons showed that $50 \%$ women were undergone emergency repeat caesarean section. According to this study out of 50 gravid women with labor pain, $16(32 \%)$ patients were delivered vaginally with spontaneous and assisted and $32(68 \%)$ by repeat emergency caesarean section. Significantly, higher number of had undergo caesarean section. A health report of Statistics Canada 1996, it was $33 \%$ [32]. Age of the study patients, 25(50\%) women belonged to 20-30 years' age group $20(40 \%)$ were in $31-40$ age group and rest $5(30 \%)$ were $<40$ years. In Sultana's study $85 \%$ women were in age group 20-30 years [33]. This is because usually maximum fertility of women was in 20-35 years' age group. This result was highly significant of unpaired ttest $(\mathrm{p}=0.001)$. The most important factor that prevents obstetricians form allowing women to undergo a vaginal delivery following a caesarean section has been the fear of uterine rupture or silent scar dehiscence. In the present study, most of the women of vaginal delivery $15(93.75 \%)$ and caesarean section 30(88.24\%) had intact uterine scar. Rupture was detected in $1(6.25 \%)$ women after vaginal delivery and $1(2.94 \%)$ after caesarean section. However, this test showed no statistically significant difference between ultimate mode of delivery. Risk of rupture was present in attempted of VBAC, whatever is the ultimate of delivery. It shown status of uterine scar in attempting VBAC. In this study, most of the women had intact uterine scar 45(90.99\%), followed by scar rupture $1(6.25 \%)$ and impending rupture $3(8.82 \%)$. The rate of the scar in attempting VBAC was $4.9 \%$, in Sultana's study [33]. In this study it was $4.5 \%$, which is similar to the study. This incidence of scar is obviously high in comparison to Lyndon Rachelle study 5.2/1000 with spontaneous onset of labor [16]. Regarding maternal and fetal outcome in all 16(100\%) women of successful vaginal delivery group survived and no maternal death. Similar findings were shown in a study by Chowdhury [26]. Though fetal outcome is the ultimate outcome of pregnancy and labor, so it is an important of the study. According to the study living fetus were 14 out of 16 , neonatal death was only 1 , which was not related to delivery and stillbirth was $1(6.25 \%)$. On the other hand, caesarean section (failed to VBAC), the living fetal were 33 out of 34 cases, stillbirth $1(6.25 \%)$ and no neonatal death. Almost equal number of babies survived in successful attempted of VBAC. This result was significant. Regarding maternal complications, out of 50 patient's hysterectomy was needed in $2(4.5 \%)$, compared to Sultana's (10\%) [33] it was all most half in percentage. Perinatal loss was $3(6 \%)$ cases, which similar to Roosmalen (7\%) study. Analyzing overall maternal complications were much less in those who were successful in attempted of VBAC $(14.5 \%)$ than those who needed CS $(85.4 \%)$. Wound infection was $5(14.21 \%)$, wound dehiscence $2(5.88 \%)$, were absolutely associated with those who failed in attempted VBAC. On the other hand, perinatal tear $1(6.25 \%)$ was completely associated with vaginal delivery, especially assisted vaginal delivery. In this study, chance of development of postpartum hemorrhage more or less same in vaginal delivery $3(18.75 \%)$ and in CS 4(11.76\%) after attempted VBAC. The exception in this study was uterus rupture, which rate was 2.5 times in failed attempted VBAC or repeat CS (34) than in successful VBAC 16 in numbers. According to the study the achievement of VBAC was significantly influenced by condition of the patient's antenatal care, gravidity (mean value $3.03 \pm 1.31$ years), parity (mean value $1.75 \pm 1.6$ ) and inter delivery interval (mean value $4.11 \pm 1.92$ years). It was not significantly influenced by fetal weight, when it was less than $3 \mathrm{~kg}$ (mean value $2.85 \pm 0.36 \mathrm{~kg}$ ). Duration of hospital stay was significantly reduced by successful vaginal birth after caesarean (VBAC), the mean value was (2.28 \pm 2.26 days).

\section{CONCLUSION}

Now a day, vaginal delivery of pregnant women with history of previous one caesarean section with non-recurrent indication was established. It has been showed that the outcome of trail of labor in past caesarean delivery is acceptable, effective and safe for both mother and fetus, if the women is properly selected. This has been possible because of modern surgical technique, safe anesthesia, facilities for blood transfusion and modern electronic equipment's for monitoring of the fetus during intra partum period. Proper counseling for trial labor and evaluation, of the cases of women with prior caesarean section has been considered a key method of reducing the caesarean section rate. In developing countries like Bangladesh it is better to give trial of labor in patients who do not have absolute contra- indications for vaginal delivery.

There are no standard guidelines for patients of previous cesarean section to attempt VBAC. There is insufficient evidence to recommend the mode of delivery in pregnancies with previous cesarean and this 
subject continues to be a matter of debate at present. Caesarean section rate has increased in Bangladesh by $16 \%$. Current CS rate is $23 \%$ of all births. This high rate need reduction to acceptable level of $15 \%$. However, profile of Bangladeshi women having repeated CS and indications are unknown. Knowledge on these issues may help plan appropriate strategies to reduce CS rate. Aim of this study is to assess profile of women who underwent repeat CS (Elective and emergency).

\section{REFERENCES}

1. Khotaba, S., Volfson, M., Tarazova, L., Odeh, M., Barenboym, R., Fait, V., ... \& Oettinger, M. (2001). Induction of labor in women with previous cesarean section using the double balloon device. Acta Obstetricia et Gynecologica Scandinavica: SHORT REPORT, 80(11), 10411042.

2. Mukherjee, S. N. (2006). Rising caesarean section rate -Review article. J obstet gynaecol India, 56:298-300.

3. SOGC. (2005). Clinical practice guidelines for VBAC Catalin S, Buhimschilrnia A, Patel BS, Andrew M, P. Weiner MCP. Rupture of the uterine scar during term labour: contractility or biochemistry? BJOG; 112:38.

4. World Health Organization. (1985). Appropriate technology for birth. Lancet, 2, 436-437.

5. British Columbia Reproductive Care Program. Obstetric guideline 8: vaginal birth after previous Caesarean birth. J Soc Obstet Gynaecol Can, 1997; 19:11258.

6. Felkin, R. W. (1884). In 1879 from his article "Notes on Labor in Central Africa" published in the Edinburgh Medical Journal, 20, 922-930

7. Naji, O., Abdallah, Y., \& Paterson-Brown, S. (2010). Cesarean birth: Surgical techniques. Glob Libr Women's Med.

8. Datta, D. C. (2011). Pregnancy with history of pevious Caesarean section. In: Textbook of obstetrics including pcrinatology and contraception. 7th ed. Calcutta: New Central Book Agency, Hiralal Konar, 327-331.7099770488

9. Dodd, J. M., Crowther, C. A., Huertas, E., Guise, J. M., \& Horey, D. (2013). Planned elective repeat caesarean section versus planned vaginal birth for women with a previous caesarean birth. Cochrane Database of Systematic Reviews, (12).

10. Ainbinder, S. W. (2003). Operative delivery. I Duchene AH, Nathan L, editors. Current obstetric and gynecologic, diagnosis and treatment. 9th ed. New York: A Lang Medical Books, 499-530.

11. Gotoh, H., Masuzaki, H., Yoshida, A., Yoshimura, S., Miyamura, T., \& Ishimaru, T. (2000). Predicting incomplete uterine rupture with vaginal sonography during the late second trimester in women with prior cesarean. Obstetrics \& Gynecology, 95(4), 596-600.

12. Zeteroglu, S., Ustun, Y., Engin-Ustun, Y., Sahin, H. G., \& Kamaci, M. (2005). Eight years' experience of uterine rupture cases. Journal of obstetrics and gynaecology, 25(5), 458-461.

13. Kayani, S. I., \& Alfirevic, Z. (2005). Uterine rupture after induction of labor in women with PCS. BJOG, 112:451.

14. McDonagh, M. S., Osterweil, P., \& Guise, J. M. (2005). The benefits and risks of inducing labour in patients with prior caesarean delivery: a systematic review. BJOG: An International Journal of Obstetrics \& Gynaecology, 112(8), 1007-1015

15. Dunn, E. A., \& O’Herlihy, C. (2005). Comparison of maternal satisfaction following vaginal delivery after caesarean section and caesarean section after previous vaginal delivery. European Journal of Obstetrics \& Gynecology and Reproductive Biology, 121(1), 56-60.

16. Hassan, A. (2005). Trial of scar and VBAC. J Ayub med coll Abbottabad, 17:57.

17. Sur, S., \& Mackenzie, I. Z. (2005). Does discussion of possible scar rupture influence preferred mode of delivery after a caesarean section?. Journal of obstetrics and gynaecology, 25(4), 338-341.

18. Macones, G. A., Peipert, J., \& Nelson, D. B. (2005). Maternal complications with vaginal birth after cesarean delivery: a multicenter study. Am J Obstetrics Gynecology, 193:1656-62.

19. Paré, E., Quiñones, J. N., \& Macones, G. A. (2005). Vaginal birth after caesarean section versus elective repeat caesarean section: assessment of maternal downstream health outcomes. Int J Gyn Obst. 89:319.

20. Porreco, R. P. (1990). Meeting the challenge of the rising cesarean birth rate. Obstetrics and gynecology, 75(1), 133-136.

21. Pridjian, G. A. B. R. I. E. L. L. A., Hibbard, J. U., \& Moawad, A. H. (1991). Cesarean: changing the trends. Obstetrics and gynecology, 77(2), 195-200.

22. Sachs, B. P., Kobelin, C., Castro, M. A., \& Frigoletto, F. (1999). The risks of lowering the cesarean-delivery rate. N Engl J Med, 340:54-7.

23. Wing, D. A., \& Paul, R. H. (1999). Vaginal birth after Cesarean section: selection and management. Clinical obstetrics and gynecology, 42(4), 836.

24. Crowther, C. A., Dodd, J. M., Hiller, J. E., Haslam, R. R., Robinson, J. S., \& Birth After Caesarean Study Group. (2012). Planned vaginal birth or elective repeat caesarean: patient preference restricted cohort with nested randomised trial. PLoS Med, 9(3), e1001192.

25. Betrán, A. P., Ye, J., Moller, A. B., Zhang, J., Gülmezoglu, A. M., \& Torloni, M. R. (2016). The increasing trend in caesarean section rates: global, regional and national estimates: 1990-2014. PloS one, 11(2), e0148343.

26. Flamm, B. L., Goings, J. R., Liu, Y., \& WoldeTsadik, G. (1994). Elective repeat cesarean delivery versus trial of labor: a prospective 
multicenter study. Obstetrics and Gynecology, 83(6), 927-932.

27. Marx, J. Wiener, N. Davies, H. (2001). A survey of the influence of patients' choice on the increase in the caesarean section rate. Journal of Obstetrics and Gynaecology, 21(2), 124-127.

28. Krishnamurthy, S., Fairlie, F., Cameron, A. D., Walker, J. J., \& Mackenzie, J. R. (1991). The role of postnatal $\mathrm{X}$ - ray pelvimetry after caesarean section in the management of subsequent delivery. BJOG: An International Journal of Obstetrics \& Gynaecology, 98(7), 716-718.

29. Paré, E., Quiñones, J. N., \& Macones, G. A. (2005). Vaginal birth after caesarean section versus elective repeat caesarean section: assessment of maternal downstream health outcomes. Int J Gyn Obst, 89:319.

30. Irvine, L. M. (2001). Maternal request for caesarean section: is it obstetrician driven?. Journal of Obstetrics and Gynaecology, 21(4),
373-374.

31. Tongson. (2003). Success rate of VBAC al Maharaj Nakorn Chiang Mai Hospital in Thailand. J Med Assoc Thai; 86:829-33.

32. Health Reports has a unique Statistics Canada catalogue number: 82-003. The English paper version is $82-003-\mathrm{XPE}$; the electronic version is 82-003-XIE.

33. Sultana, S. (2000). A study on outcome of postCaesarean pregnancy at Chittagong Medical College Hospital [dissertation]. Dhaka: Bangladesh College of Physicians and Surgeons. 39-40.

34. ACOG. (2002). Introduction of labor for vaginal birth after Caesarean section. Obetet Gyneco, 1; 99:679.

35. MMWR. (2002). VBAC-California 1996-2000. MMWR, Center for Disease Control and Prevention, 51:996-9. 